Check for updates

Cite this: RSC Adv., 2017, 7, 30717

Received 26th February 2017

Accepted 1st June 2017

DOI: $10.1039 / \mathrm{c} 7 \mathrm{ra02384g}$

rsc.li/rsc-advances

\section{Bovine serum albumin-loaded nano-selenium/ICG nanoparticles for highly effective chemo-photothermal combination therapy $\dagger$}

Guanjun Deng, ${ }^{\text {ab }}$ Ting Zhu, ${ }^{\text {ad }}$ Lihua Zhou, ${ }^{\text {ac }}$ Jingnan Zhang, ${ }^{a}$ Sanpeng Li, ${ }^{\text {ab }}$ Zhihong Sun, ${ }^{a}$ Jiazheng Lai, ${ }^{a}$ Xiaoqing Meng, ${ }^{\text {ab }}$ Wenjun Li, ${ }^{a}$ Pengfei Zhang, ${ }^{a}$ Yayun $\mathrm{Wu}^{\mathrm{a}}{ }^{\mathrm{T}} \mathrm{Tao}$ Jiang, ${ }^{\mathrm{a}}$ Dapeng Ni, ${ }^{\text {ab }}$ Weiqiang Yan, ${ }^{9}$ Mingbin Zheng, ${ }^{\text {aef }}$ Ping Gong ${ }^{* a e f}$ and Lintao Cai (D)*a

A combination of chemotherapy and photothermal treatment has already become a promising strategy for cancer treatment. Hence, it is highly desirable to develop a safe and effective delivery system. In the present study, we have developed bovine serum albumin-loaded nano-selenium (SeNPs)/indocyanine green (ICG) nanoparticles (BSINPS) that were generated by a programmed assembly method. They could simultaneously deliver SeNPs (a chemotherapeutic agent) and ICG (a photothermal agent) to tumor regions for combined chemo-photothermal therapy. BSINPs presented good monodispersity, a suitable size, fluorescence stability, and characteristic spectral features in comparison with free ICG. Moreover, the BSINPs displayed a longer retention time in tumors and a higher temperature response under laser irradiation. In addition, the process of subcellular location in vitro and the distribution of BSINPs in vivo could be monitored. The combined therapy offered by the BSINPs with laser irradiation synergistically induced the death of U87L glioma cells, and completely inhibited U87L tumor growth in vivo compared with chemo or photothermal therapy alone. Outstandingly, no tumor recurrence or treatment-induced toxicity was observed after a single-dose of BSINPs with laser irradiation. The results indicate that the well-defined BSINPs are highly desirable for cancer treatment with chemo-photothermal therapy.

\section{Introduction}

Cancer is the most common cause of death all over the world. The standard treatments for cancer which rely on a single therapeutic treatment remain unsatisfactory. Combination therapy is the co-administration of two or more different drugs or treatments for a patient. It is a normal clinical practice in the therapy for many types of cancer. These combination therapy regimens are tending to achieve therapeutic synergy; their

\footnotetext{
${ }^{a}$ Guangdong Key Laboratory of Nanomedicine, CAS Key Lab for Health Informatics, Shenzhen Institutes of Advanced Technology, Chinese Academy of Sciences, Shenzhen 518055, P. R. China.E-mail: lt.cai@siat.ac.cn; ping.gong@siat.ac.cn ${ }^{b}$ University of Chinese Academy of Sciences, Beijing 100049, P. R. China 'College of Life Sciences, Hunan Normal University, Changsha 410081, P. R. China ${ }^{a}$ Shenzhen Second People's Hospital, The First Affiliated Hospital of Shenzhen University, Shenzhen 518037, P. R. China

${ }^{e}$ Guangdong Key Laboratory for Research and Development of Natural Drugs, Guangdong Medical University, Dongguan 523808, China

${ }^{f}$ State Key Laboratory of Chemo/Biosensing and Chemometrics, Hunan University, Changsha 410082, P. R. China

${ }^{g}$ Department of Radiology, Peking University Shenzhen Hospital, Shenzhen 518036, P. R. China

$\dagger$ Electronic supplementary information (ESI) available: Experimental details, Fig. S1-S3. See DOI: 10.1039/c7ra02384g
}

medicinal effect is greater than that of a single drug or treatment. ${ }^{1-3}$ It was reported that a co-delivery system loaded with two chemotherapeutics (doxorubicin and 5-fluorouracil) acted through different anticancer mechanisms, which indicated the potential of combination therapy and improved the efficiency of prostate cancer treatment. ${ }^{4}$ A co-delivery strategy utilizing rapamycin (an antiangiogenic drug) and cisplatin significantly induced apoptosis in human melanoma cells and inhibited remarkably the growth of melanoma compared to either singleagent regimen. ${ }^{5}$ It is also worth combining the treatments of near infrared (NIR) photothermal therapy (PTT) and chemotherapy. ${ }^{6}$ PTT involves the employment of photothermal agents, such as gold nanomaterials, carbon nanotubes and indocyanine green (ICG) which localize in tumors and strongly absorb NIR light, converting it into cytotoxic heat and leading to irreversible damage in tumor cells. ${ }^{7-10}$ PTT, as a non-invasive treatment, is an attractive alternative to standard therapy for cancer, because of its maneuverability and low side effects. ${ }^{11}$

Selenium (Se) is an essential trace element with important physiological and pharmacological functions. It is also vital to intracellular redox states, the immune system and life span. ${ }^{12-16}$ Different concentrations of selenium have different pharmacological actions. At low concentrations, selenium can get rid of reactive oxygen species (ROS) and the seleno-enzymes can 
repair damaged DNA, and thus it can protect cell. At high concentrations, selenium can induce the production of ROS, leading to cell apoptosis and cell death. ${ }^{17-19}$ The antioxidant property of selenium has been well-studied for many years, in a mechanism catalyzed by glutathione peroxidase. ${ }^{\mathbf{2 0 , 2 1}}$ Recently some scholars have begun to pay attention to the pharmacological action of selenium, because some forms of selenium generate ROS resulting in oxidative stress and cell death. ${ }^{22,23}$ Thus selenium serves as a new chemotherapeutic agent for cancer treatment. The role of selenocompounds as sensitizers can suppress many cancer cells and prevent the occurrence of drug resistance in cancer cells, and it is considered that selenium plays an important role in cancer prevention. ${ }^{24-27}$ In addition, zero-valent elemental selenium nanoparticles (SeNPs) have a high Se-density formulation with the potential for local delivery of high doses into tumor cells when compared with other selenium forms. ${ }^{28}$ It has been reported that selenium nanoparticles could significantly suppress malignant mesothelioma. ${ }^{29}$

In this study, we developed biodegradable bovine serum albumin (BSA)-loaded nano-selenium/ICG nanoparticles (BSINPs) with the simultaneous incorporation of chemotherapeutic and photothermal agents for in vitro/in vivo tumor targeting therapy. Herein, a programmed assembly method was developed for fabricating the BSINPs. BSA served as the template and as coating molecules to induce the formation of SeNPs through biomineralization in reduction conditions, and then ICG was modified on the surface of the nanoparticles, to obtain multicomponent BSINPs. The BSINPs are a dualfunction agent with the capability for optical imaging and an antitumor effect. The fluorescence (FL) of ICG in BSINPs could be used to monitor the process of subcellular location in vitro and the distribution of particles in vivo. The chemophotothermal combination therapy of BSINPs not only induced U87L cell death in vitro but also offered a significantly improved anticancer effect in vivo and led to tumor eradication without any recurrence. Hence, the combination therapy of BSINPs has great potential in cancer therapy.

\section{Results and discussion}

\section{Characterization of the BSINPs}

The synthetic process for the preparation of BSINPs is described in Fig. 1. SeNPs were synthesized by reducing sodium selenite $\left(\mathrm{Na}_{2} \mathrm{SeO}_{3}\right)$ with glutathione (GSH) through a selenodiglutathione intermediate (GSSeSG) which could release elemental selenium at alkaline $\mathrm{pH}^{.0,31}$ SeNPs generated reactive oxygen species, resulting in oxidative stress and cell death. ${ }^{32}$ Thus selenium served as a new chemotherapeutic agent for cancer treatment. BSA was used as a colloidal stabilizing agent for the SeNPs. ICG dye molecules were covalently conjugated to the surface of the SeNPs.

Firstly, the morphology and particle size of the BSINPs were examined by transmission electron microscopy (TEM) (Fig. 2a). The particles are generally spherical in shape and the average diameter is about $40 \mathrm{~nm}$, which indicates the successful assembly of the BSINPs; the size of a single SeNP in the core of the BSINPs is about $20 \mathrm{~nm}$ (Fig. S1 $\dagger$ ). ${ }^{33,34}$ BSINPs exhibit good

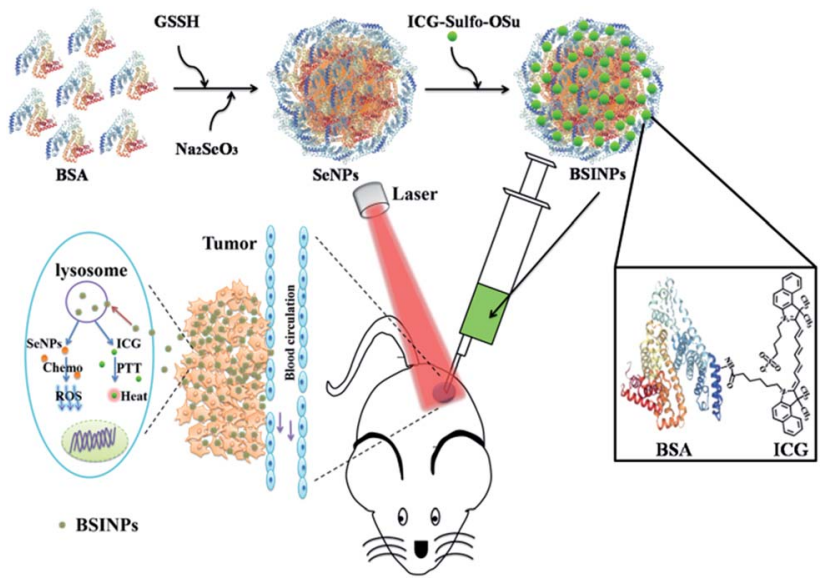

Fig. 1 Schematic diagram for the preparation and chemo-photothermal combination therapy of BSINPs.

monodispersity and size distributions with an average hydrodynamic diameter of $78 \pm 1.5 \mathrm{~nm}$ (obtained by dynamic light scattering; DLS) (Fig. 2b). The size distribution showed that the BSINPs were about $100 \mathrm{~nm}$, indicating that they should offer an enhanced permeability and retention effect (EPR), and could improve drug delivery to tumors. ${ }^{35}$ In addition, the zeta potential of the BSINPs was about $-22.2 \mathrm{mV}$.

The UV/vis absorption and FL spectra displayed that BSINPS have the characteristic absorption band and emission peak of ICG dye (Fig. 2c and d), which verified the existence of ICG dye in the BSINPs. Compared with free ICG, the absorption and emission peaks of BSINPs were red-shifted $16 \mathrm{~nm}$ to $796 \mathrm{~nm}$ and $10 \mathrm{~nm}$ to $817 \mathrm{~nm}$, respectively, indicating that BSINPs can impact the optical properties of ICG. The structure of the BSINPs was further characterized by FT-IR and XPS. As shown in Fig. S2, $\uparrow$ the peak of ICG at $1080 \mathrm{~cm}^{-1}$ exists in the FT-IR mapping of the BSINPs, and Fig. S3† shows that elemental selenium was found in SeNPs and BSINPs. As shown in Fig. S4a, $\uparrow$ the FL stability of the ICG in BSINPs was higher than that of free ICG, which suggests that BSINPs could improve the FL stability of ICG. The particle sizes of the BSINPs remained around the initial particle size without aggregation or precipitation over 4 weeks, as shown in Fig. S4b and c. $\uparrow$ The high stability of the BSINPs may be attributed to BSA which is a good colloidal stabilizing agent and can improve the BSINPs' biocompatibility and stability.

To evaluate the photothermal efficiency of the BSINPs, temperature changes under laser irradiation were detected by an infrared thermal imaging camera. The temperature of BSINPs, free ICG, SeNPs and PBS rose under irradiation at $0.8 \mathrm{~W}$ $\mathrm{cm}^{-2}$ for $8 \mathrm{~min}$. The maximum temperatures of BSINPs and free ICG reached $58.2{ }^{\circ} \mathrm{C}$ and $56.5{ }^{\circ} \mathrm{C}$, respectively, while that of the SeNPs and PBS hardly changed (Fig. 2e). When the temperature of the BSINPs exceeds $43{ }^{\circ} \mathrm{C}$, it will lead to irreversible damage to tumor cells. The temperature increase of the BSINPs was higher than that of free ICG, and the result was similar to that obtained in a previous study of ICG-containing nanoparticles which were more efficient in producing a laser-dependent 


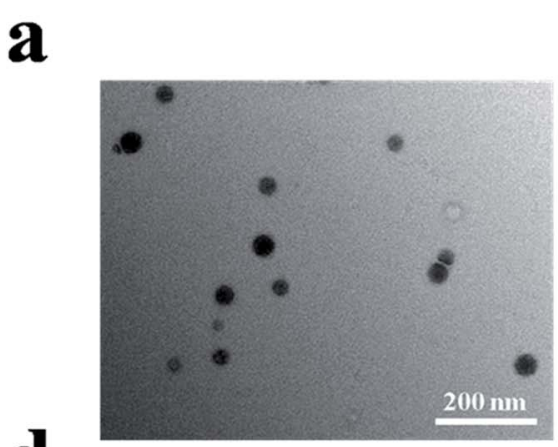

b
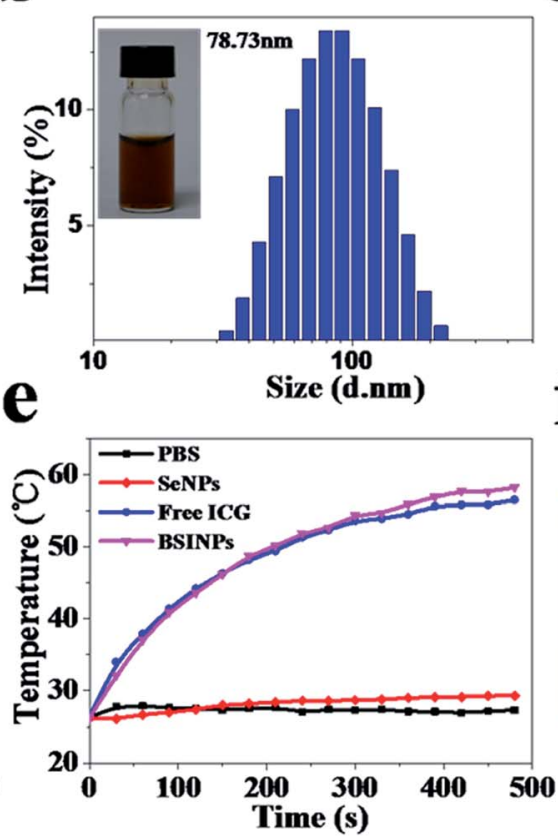

c

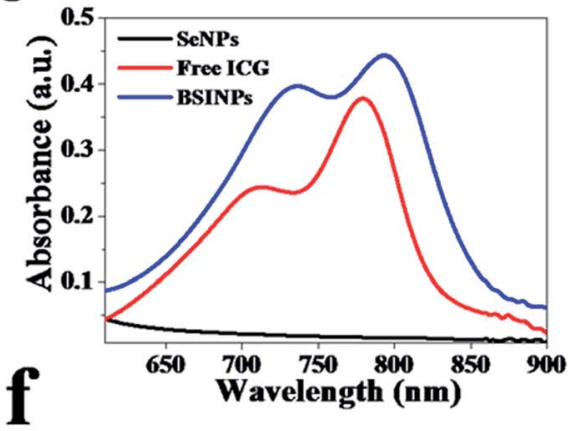

PBS SeNPs Free ICG BSINPs

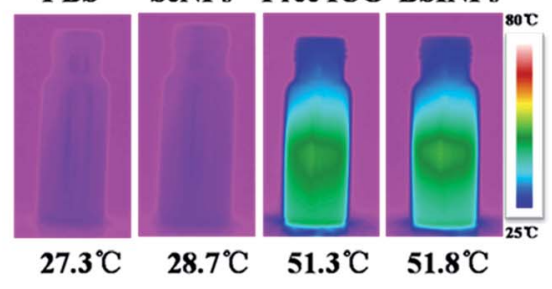

Fig. 2 Characterization of the BSINPs. (a) TEM images of BSINPs. (b) Size distribution of BSINPs. (c) UV/vis absorption spectra of free ICG, SeNPs and BSINPs. (d) FL spectra of free ICG, SeNPs and BSINPs. (e) Temperature elevation profiles of free ICG, SeNPs and BSINPs. (f) Infrared thermographic maps of tubes containing free ICG, SeNPs and BSINPs measured 5 min after continuous laser irradiation.

temperature rise than free ICG. ${ }^{36}$ A possible reason is that the BSINPs have a higher ICG-density concentration than the free ICG.

In vitro cellular uptake of BSINPs

The cell uptake of BSINPs and free ICG was investigated through confocal microscopy. After $4 \mathrm{~h}$ incubation, a great number of BSINPs had entered into U87L glioma cells, while only a small amount of free ICG was observed in these cancer cell (Fig. 3a and $55 \dagger$ ). The quantitative results of flow cytometry analysis displayed that the average FL intensity of the BSINPs was almost 10-fold more than that of free ICG (Fig. 3b), and Fig. S6 and S7† showed the endocytosis of BSINPs in lysosomes. The results indicated that the subcellular localization of the a
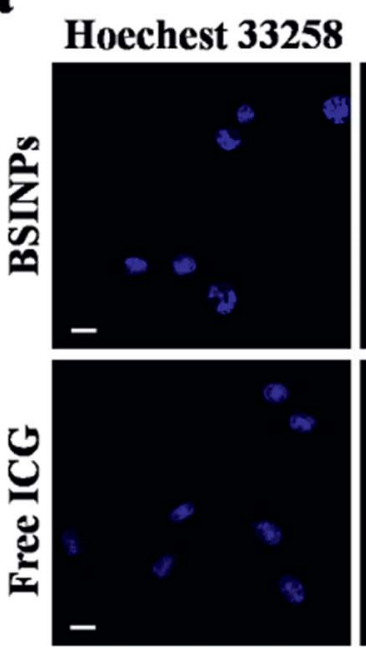
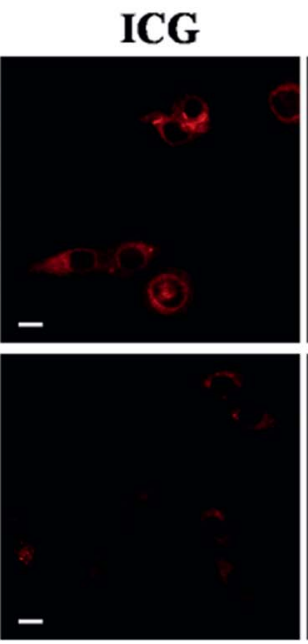

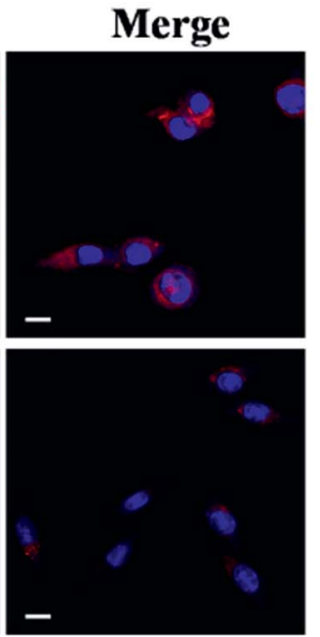

b

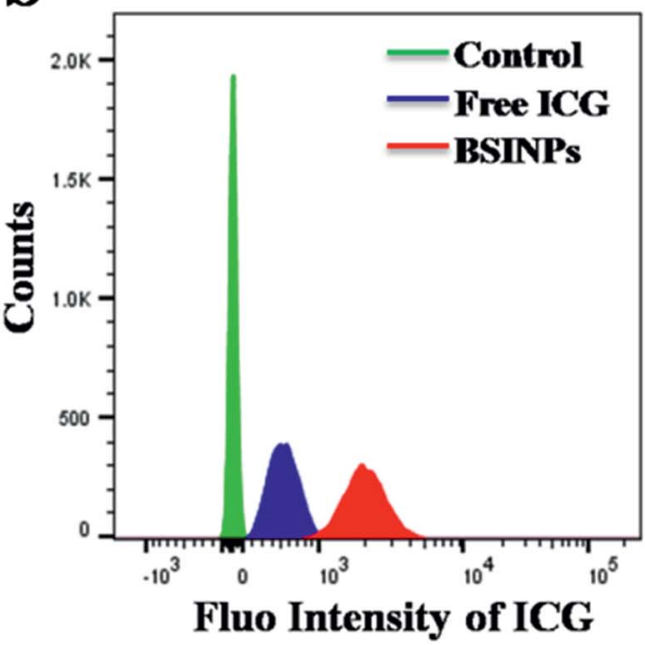

Fig. 3 The cell uptake of BSINPs. (a) Confocal FL images of U87L cells incubated with $10 \mu \mathrm{g} \mathrm{mL}^{-1}$ free ICG and BSINPs (containing $10 \mu \mathrm{g} \mathrm{mL}{ }^{-1}$ ICG) for $4 \mathrm{~h}$. Blue represented FL of Hochest 33258, and red represented FL of ICG. (b) Flow cytometric analysis of U87L cells incubated with media (green), free ICG (blue), BSINPs (red) for $4 \mathrm{~h}$. 

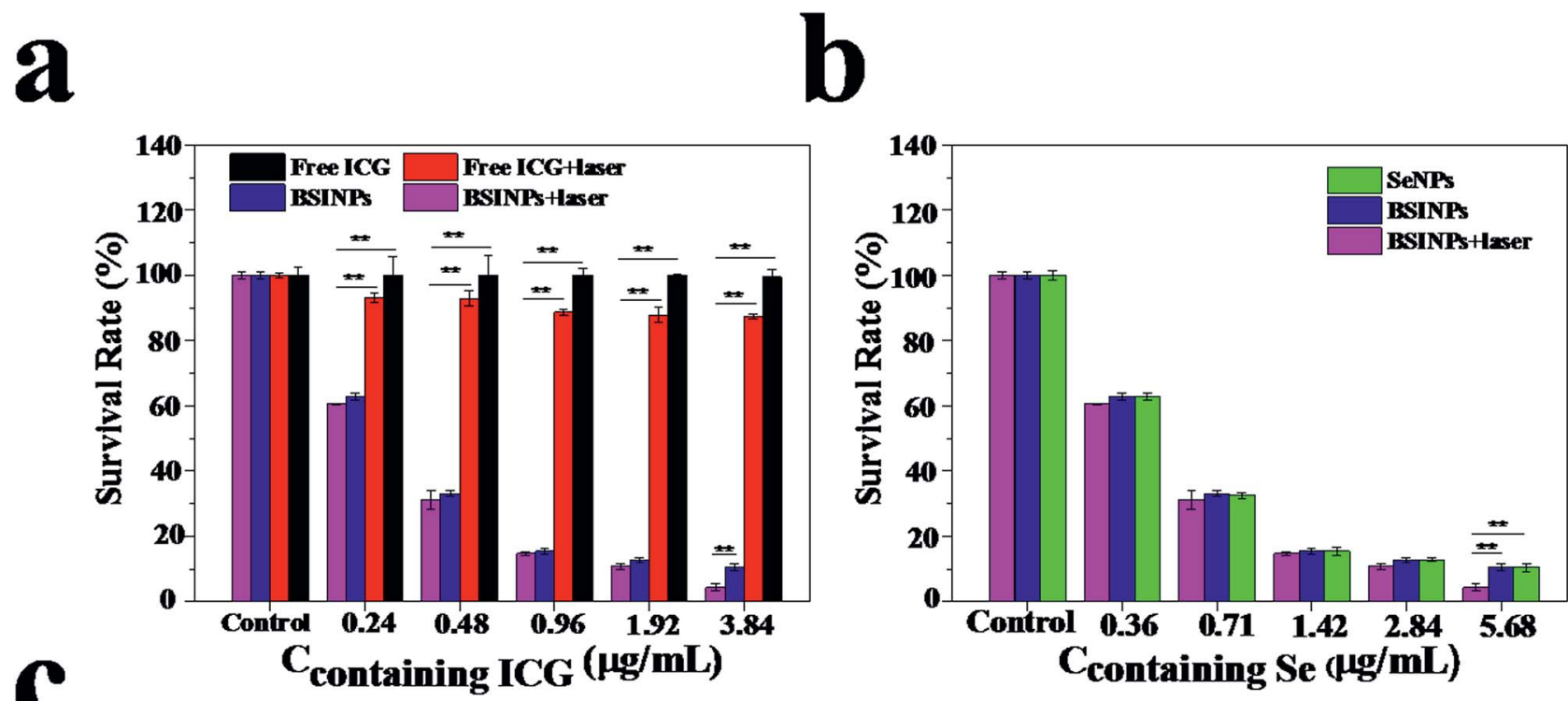

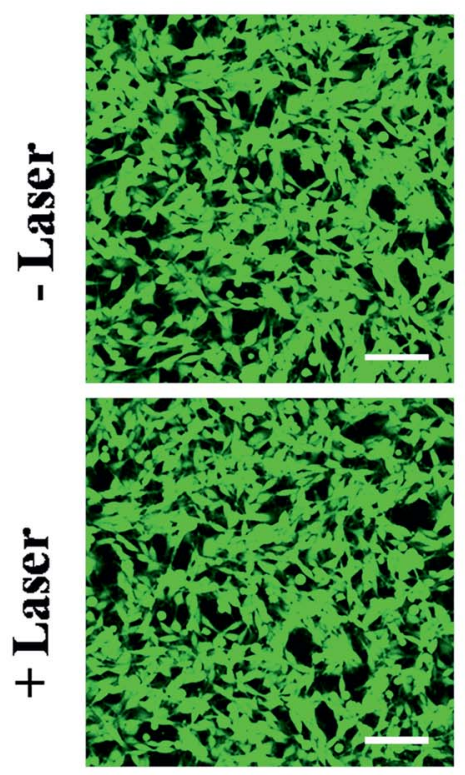

Control

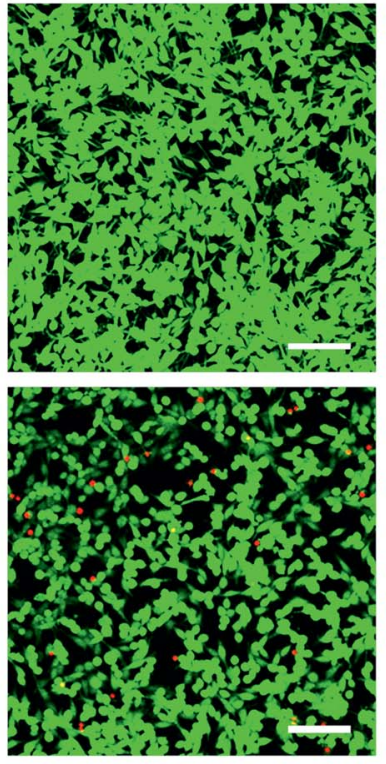

Free ICG

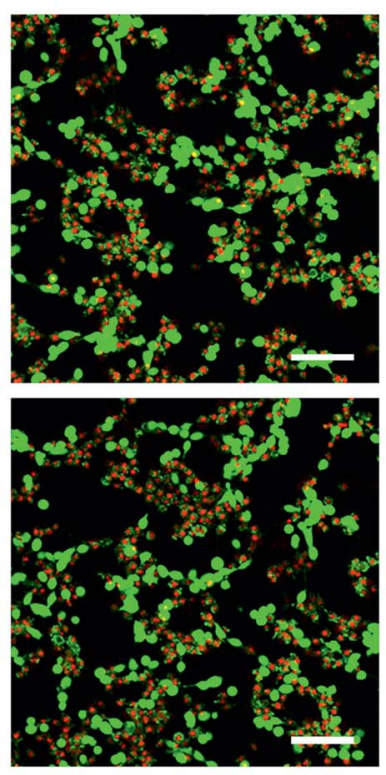

SeNPs
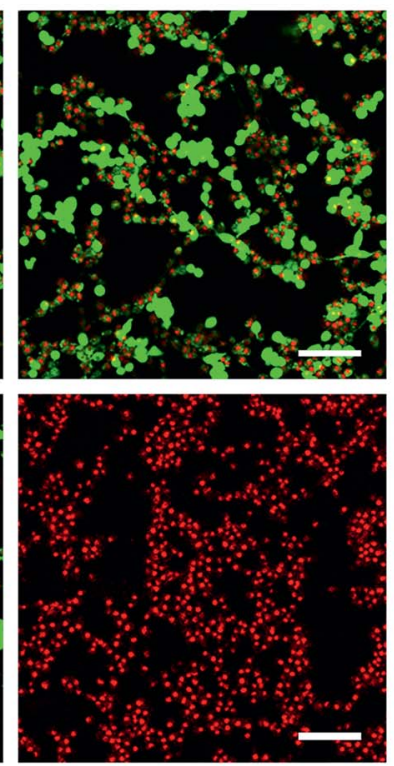

BSINPs

Fig. 4 The chemo-photothermal treatments in U87L cells. (a) and (b) Cell survivals of U87L after treatment using free ICG, SeNPs or BSINPs with different concentration under $808 \mathrm{~nm}$ laser irradiation $(0.8 \mathrm{~W} \mathrm{~cm}-2808 \mathrm{~nm}$ laser, $5 \mathrm{~min})$. (c) FL images of U87L cells after chemo-photothermal treatment. Viable cells were stained green with calcein-AM, and dead/later apoptosis cells were stained red with PI. (Scale bar, $100 \mu \mathrm{m}$ ).

BSINPs is in the lysosome and that the use of BSA could improve the cell uptake capability for ICG. The raised cell uptake of BSINPs contributed to improving the chemotherapeutic and phototherapeutic efficiency.

\section{In vitro chemotherapy and photothermal therapy}

Since SeNPs have a chemotherapeutic effect, BSINPs could serve as a dual-functional agent for chemotherapy and photothermal therapy. Firstly, the cytotoxic effect of BSINPs on U87L glioma cells under irradiation with a $0.8 \mathrm{~W} \mathrm{~cm}^{-2} 808 \mathrm{~nm}$ laser was investigated by CCK-8 assay. Fig. $4 \mathrm{a}$ and b demonstrates that chemotherapy alone (SeNPs) and photothermal therapy alone (free ICG + laser) caused up to $89.36 \%$ and $12.67 \%$ cell death respectively, while the combined chemotherapy and photothermal therapy of BSINPs induced cell death of up to $95.52 \%$ when the concentration of ICG contained was $3.84 \mu \mathrm{g} \mathrm{mL} \mathrm{m}^{-1}$. The result suggests that the SeNPs had a good chemotherapy effect and that the amount of ICG in the BSINPs was sufficient for photothermal treatment.

The cytotoxic effect of BSINPs on U87L glioma cells under irradiation with the $0.8 \mathrm{~W} \mathrm{~cm}^{-2} 808 \mathrm{~nm}$ laser was further investigated by confocal microscopy. The cells were labeled with calcein-AM and propidium iodide (PI) to identify live and dead/ late apoptotic cells, respectively. As shown in Fig. 4c, the cells all displayed green fluorescence in the control group, suggesting that the laser irradiation alone without nanoparticles could not 
kill U87L cells at the power density of $0.8 \mathrm{~W} \mathrm{~cm}^{-2}$. It further demonstrated that the chemotherapy and photothermal combination therapy improved the therapeutic efficiency against tumor cells.

\section{In vivo imaging and biodistribution}

In order to deliver drugs into tumor tissues, nanoparticles must enter into the tumor vasculature and interstitium. The drug nanoparticles need to stay in the tumor tissues and the drug must diffuse into the cancer cells. ${ }^{37}$ To investigate the accumulation of BSINPs in tumor tissues, the distribution of the nanoparticles in the body were measured by in vivo FL imaging of athymic nude mice with subcutaneous U87L glioma xenografts. Fig. 5a and b show the ICG FL signals and intensity distributions around the tumors after intratumoral injections of free ICG or BSINPs. The FL signals were measured in the whole bodies of the mice but the peritumor signals were rather weak because of the relatively low doses of ICG. The ICG FL intensity of the BSINPs around the tumor at 6,12 , and 24 h postinjection decreased to $98.84 \%, 93.07 \%$, and $81.41 \%$ of the initial FL intensity. In the control group (free ICG), the corresponding outcomes were respectively $46.38 \%, 33.25 \%$, and $26.75 \%$. The results suggested that the ICG FL intensity of the BSINPs did not change much for $24 \mathrm{~h}$ compared to that of the free ICG and this meant that the BSINPs were significantly retained in the tumor even after $24 \mathrm{~h}$. This was because the nanoparticles had an enhanced EPR effect, and the BSINP encapsulation reduced ICG degradation. The FL intensity of free ICG was rapidly reduced due to FL quenching of the free ICG in the physiological surroundings with its rapid aggregation and clearance by the body's metabolism. ${ }^{38}$ Fig. $5 \mathrm{c}$ and d show a comparison of the distributions of the free ICG and BSNIPs in the major organs and tumors; ICG accumulated in the liver and tumor $24 \mathrm{~h}$ after intratumoral injection. The BSINP
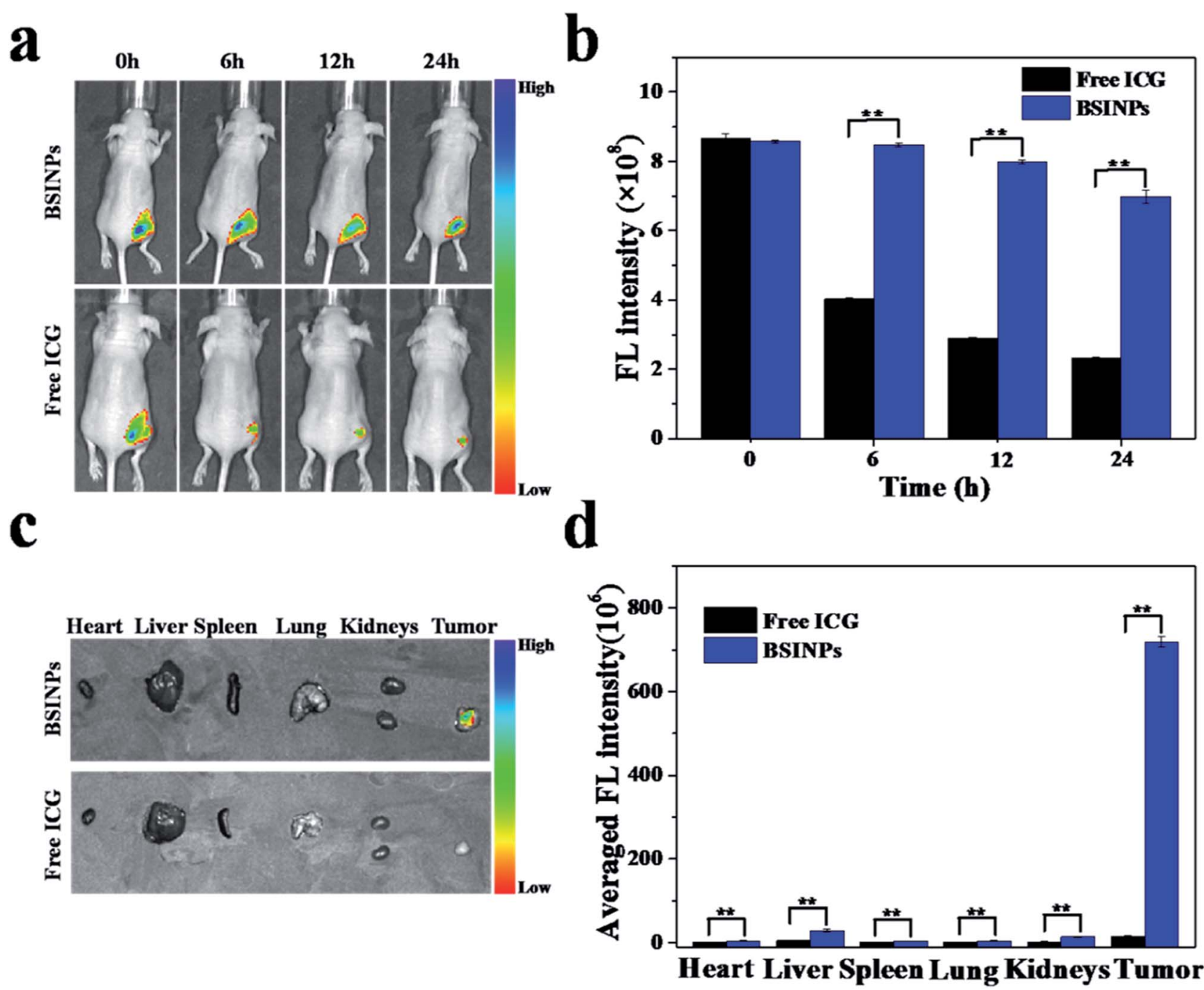

Fig. 5 In vivo imaging of nude mice bearing U87L tumors and biodistribution of ICG after intratumoral injection with free ICG or BSINPs. (a) Representative NIR FL images of U87L tumor-bearing mice after intratumoral injection with free ICG or BSINPs at different time points. (b) NIR FL intensities around the tumors quantified at the indicated time points. (c) NIR FL images of major organs and tumors after injection with free ICG or BSINPs at $24 \mathrm{~h}$. (d) Semiquantitative biodistribution of free ICG and BSINPs in nude mice as determined by the averaged FL intensity in the organs and tumors. Mean data are shown (SD $(n=3) ;(*) p<0.05,(* *) p<0.01)$. 
formulation remarkably increased the accumulation of ICG in the tumor, followed by the liver and kidneys. The BSINP averaged FL intensity in the tumor was more than 46 times that of free ICG at $24 \mathrm{~h}$ post-injection, which suggested that BSINPs had high tumor accumulation. These data are consistent with the reported pharmacokinetics assay except that ICG showed liver-specific distribution and particulates were taken up by the reticuloendothelial system (RES) in circulation. ${ }^{39}$

\section{In vivo chemo-photothermal combination therapy}

As a result of the treatment results in vitro, we applied BSINPs for in vivo cancer therapy. The real-time temperature variation of mice was measured by using an infrared thermal camera. The intratumoral temperatures were determined while the tumor regions which had been injected with PBS, free ICG or BSINPs were irradiated with a $0.8 \mathrm{~W} \mathrm{~cm} \mathrm{~cm}^{-2}$ NIR laser for $5 \mathrm{~min}$ (Fig. 6a and b). After 5 min of laser irradiation, the tumors which had been injected with free ICG or BSINPs reached temperatures of $57.1^{\circ} \mathrm{C}$ and $59.2^{\circ} \mathrm{C}$, respectively, which exceeded the threshold required to cause damage. The control group, however, exhibited only a mild temperature increase to $38.9{ }^{\circ} \mathrm{C}$, which was insufficient to irreversibly heat-damage the tumor tissue.

To further investigate the anticancer effect of the BSINPmediated chemo-photothermal combination therapy in vivo, U87L tumor tissues were stained with hematoxylin and eosin. As shown in Fig. 6c, the tumors treated with free ICG plus laser irradiation or BSINPs plus laser irradiation displayed typical characteristics of thermal damage, such as coagulative necrosis and significant karyolysis in tumors, as described previously. ${ }^{36}$

We investigated the anticancer effect of the chemophotothermal therapy in nude mice bearing U87L tumors. The representative pictures and the growth of U87L tumors reflecting the tumor size variations are displayed in Fig. 7a and b. The tumors treated with PBS or PBS plus laser irradiation grew rapidly, which showed that the U87L tumor growth was not affected by laser irradiation. Free ICG plus laser irradiation had a slight tumor-suppressing effect. Although SeNPs can offer a chemotherapeutic effect, this was insufficient to inhibit tumor growth in vivo. SeNPs usually demand multiple high dosages to obtain a satisfactory anticancer effect. Remarkably, the growth

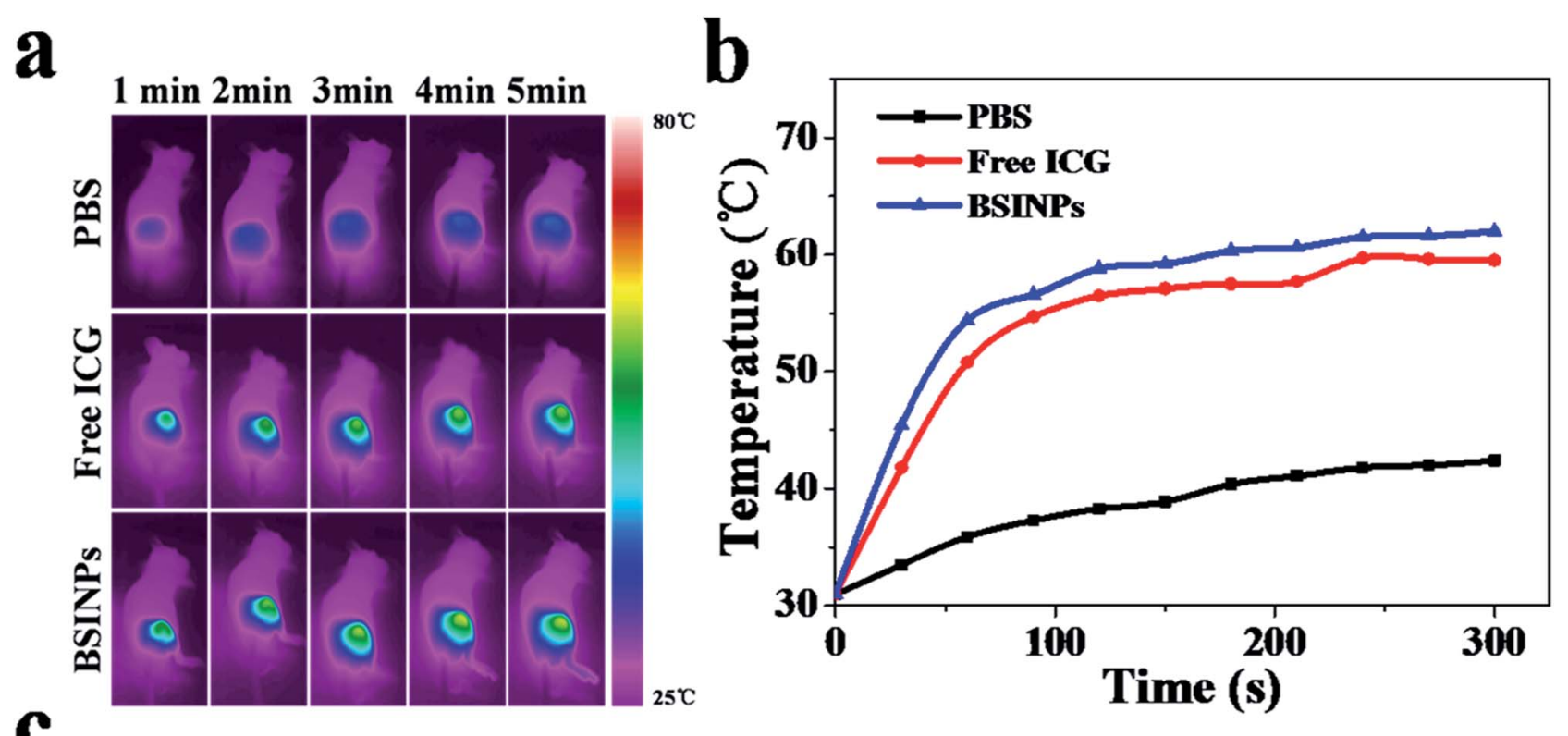

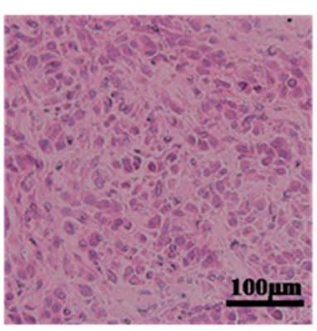

PBS

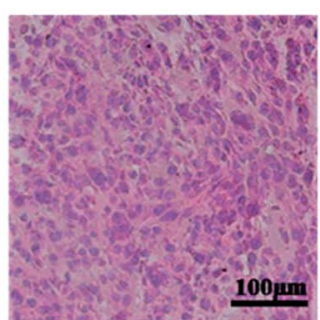

PBS+laser

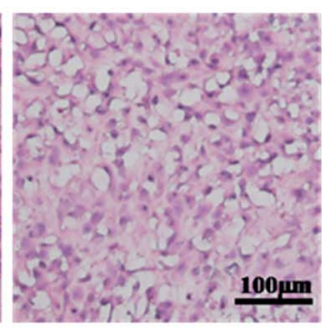

SeNPs

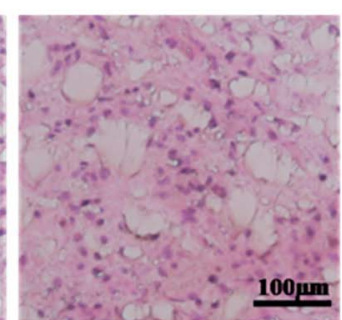

Free ICG

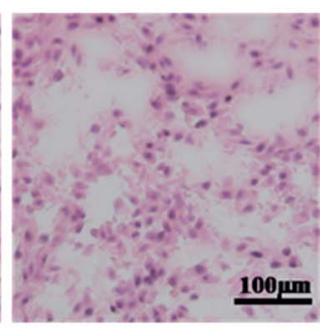

BSINPs

Fig. 6 Profiles of the temperature rises in tumor-bearing mice after intratumoral injections. (a) Representative thermal images of U87L tumorbearing mice exposed to an $808 \mathrm{~nm}$ laser $\left(0.8 \mathrm{~W} \mathrm{~cm}^{-2}, 5 \mathrm{~min}\right)$ after intratumoral injection with $100 \mu \mathrm{L} \mathrm{PBS,} 48 \mu \mathrm{g} \mathrm{mL}$ free ICG or BSINPs containing $48 \mu \mathrm{g} \mathrm{mL}^{-1} \mathrm{ICG}$. (b) The corresponding temperature rise profiles at the tumor sites during 5 min laser irradiation for mice treated with BSINPs, free ICG or PBS only. (c) Histological staining of the excised tumors $24 \mathrm{~h}$ after administration of PBS, PBS + laser, SeNPs, free ICG + laser or BSINPs + laser; the laser treatment groups were irradiated with the $808 \mathrm{~nm}$ laser at $0.8 \mathrm{~W} \mathrm{~cm}^{-2}$ for $5 \mathrm{~min}$. 
a
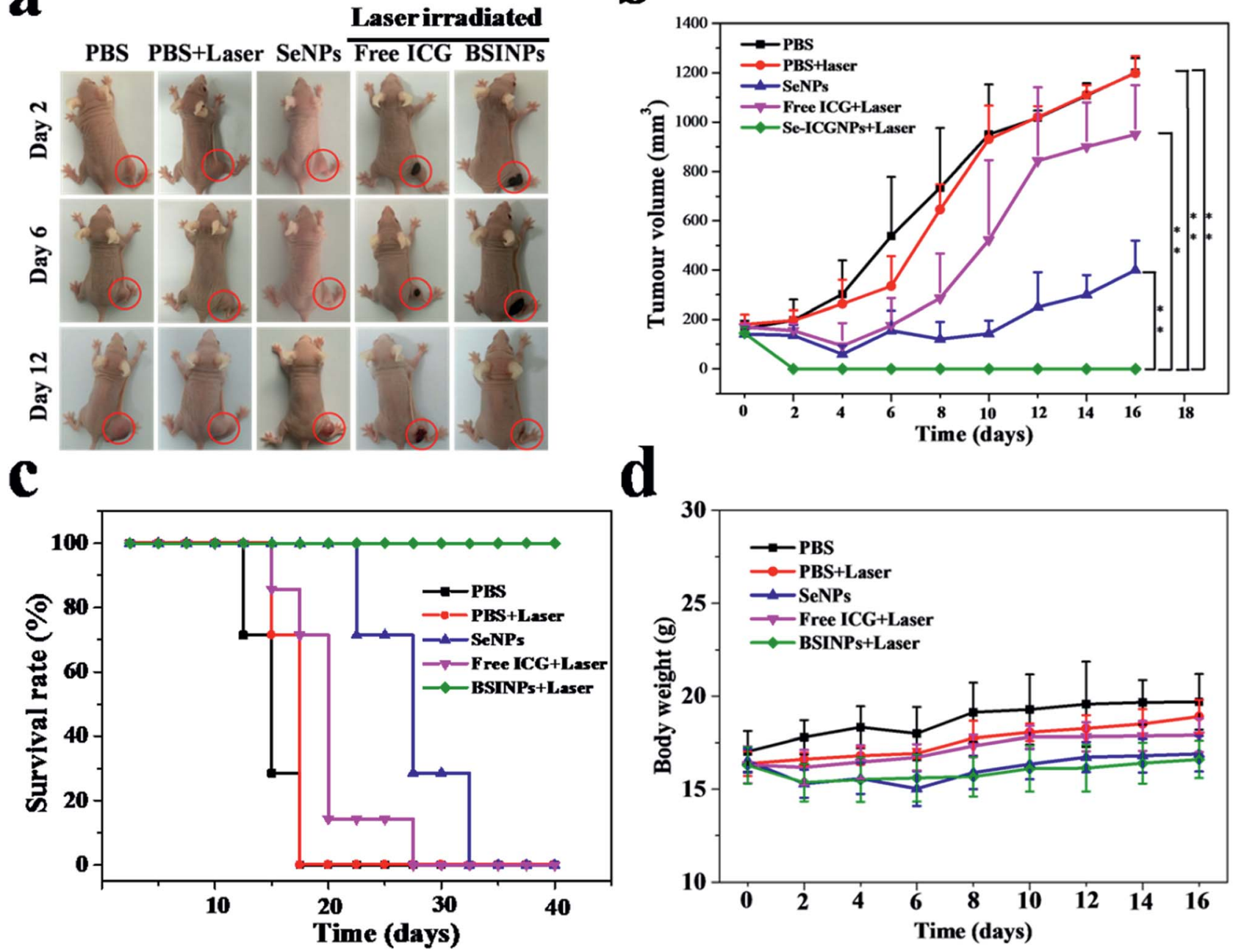

d

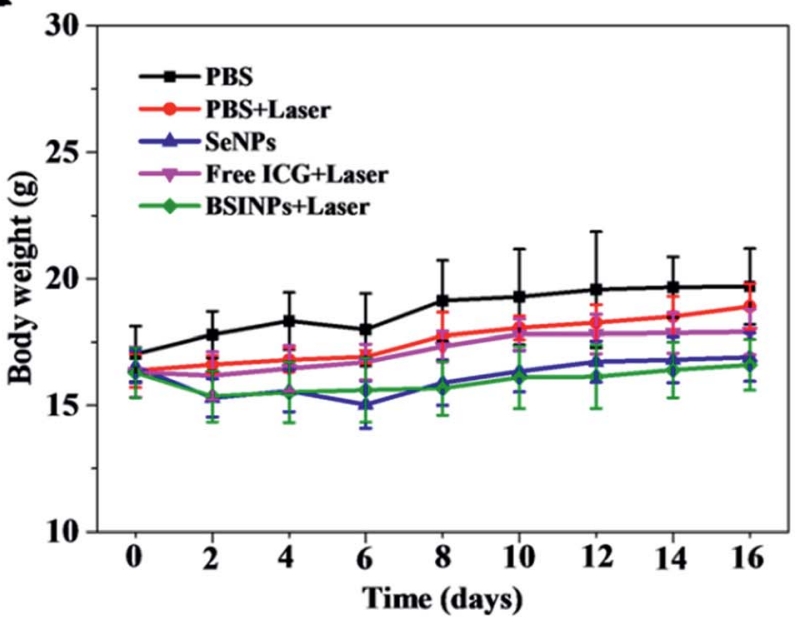

Fig. 7 In vivo chemo-photothermal therapy of BSINPs. (a) Representative photos of mice bearing U87L tumors. The tumors were marked with red circles. The treatment of BSINPs yielded the effect of combination therapy and no tumor recurrence was noted over a course of 16 days. (b) The growth curve of U87L xenograft tumors within 16 days in different groups $(n=7)$. (c) Survival rates of xenograft mice bearing U87L tumors within $40 \mathrm{~d}$ after different treatments. (d) Body weights change of mice under the different conditions. Dates indicate means and standard errors. $\left.{ }^{*}\right) p<0.05,\left({ }^{* *}\right) p<0.01$.

of U87L tumors treated with BSINPs plus laser irradiation was completely ablated by day 20. Moreover, there was no tumor recurrence observed in this group during the next 40 days. The data indicate that the simultaneous and synergetic chemotherapy and photothermal therapy mediated by the BSINPs with laser irradiation could significantly improve the anticancer effect.

The survival rate of mice with U87L tumors after treatment with BSINPs plus laser irradiation was $100 \%$ as shown in Fig. 7c. The survival rate of the SeNPs group was $71.43 \%$ on day 20 posttreatment. For mice treated with free ICG plus laser irradiation, the tumor did not recur in its original position (black scar), while the tumor grew rapidly in the tissue surrounding the scar. The survival rate of this group was $14.29 \%$ on day 20 posttreatment.
During the treatments, the body weight of the mice was monitored, as a measure of the treatment-induced toxicity (Fig. 7d). The weight of the groups treated with PBS and PBS plus laser irradiation in nude mice bearing U87L gradually increased from $4 \%$ to $11 \%$ during 16 days, and those treated with free ICG plus laser irradiation, SeNPs or BSINPs plus laser irradiation increased their body weights respectively by $9.21 \%$, $7.40 \%$ and $5.10 \%$, these groups were not significantly different from the control group, which indicated that these treatments were reasonably well-tolerated.

Furthermore, the images of H\&E-stained major organs collected from the group treated with BSINPs plus laser irradiation showed that obvious damage and inflammation were not observed compared to the control group (Fig. S8 $\dagger$ ). The results suggest that the BSINPs have low toxicity, and are suitable for simultaneous chemotherapy and photothermal therapy. 


\section{Conclusions}

We have successfully fabricated well-defined BSINPs using a programmed assembly method. The BSINPs could simultaneously deliver SeNPs and ICG to tumor regions for combined chemo-photothermal therapy. The BSINPs significantly enhanced ICG stability, produced a higher localized temperature than free ICG after laser irradiation, and effectively promoted cellular uptake of ICG. This combination of chemophotothermal therapies not only induced U87L cell death in vitro, but also suppressed U87L tumor growth and prevented tumor recurrence in vivo. Hence, the combined treatment of BSINPs plus laser irradiation could be a promising strategy for chemo-photothermal cancer therapy.

\section{Acknowledgements}

This work was supported by the National Natural Science Foundation of China (21375141, 81671758, 31571013, 51502333, 81401509, 21404115 and 81501580), the Key International S\&T Cooperation Project (2015DFH50230), the Instrument Developing Project of CAS (YZ201439), the Guangdong Natural Science Foundation of Research Team (2016A030312006), the Shenzhen Science and Technology Program (JSGG20160331185422390, JCYJ20150403091443298, KQCX20140521115045447, JCYJ20160429191503002), and the Hong Kong, Macao and Taiwan Cooperation Project of the Ministry of Science and Technology (2015DFH50230).

\section{References}

1 J. Ferlay, H. R. Shin, F. Bray, D. Forman, C. Mathers and D. M. Parkin, Int. J. Cancer, 2010, 127, 2893-2917.

2 J. Woodcock, J. P. Griffin and R. E. Behrman, N. Engl. J. Med., 2011, 364, 985-987.

3 J. Jia, F. Zhu, X.-H. Ma, Z.-W. Cao, Y.-X. Li and Y.-Z. Chen, Nat. Rev. Drug Discovery, 2009, 8, 111-128.

4 Q.-Q. Yang, Y. Yang, L. Li, W. Sun, X. Zhu and Y. Huang, ACS Appl. Mater. Interfaces, 2015, 7, 6661-6673.

5 S.-T. Guo, C. M. Lin, Z.-H. Xu, L. Miao, Y.-H. Wang and L. Huang, ACS Nano, 2014, 8, 4996-5009.

6 J. Overgaard, Cancer Res., 1976, 36, 3077-3081.

7 M. C. Chen, Z. W. Lin and M. H. Ling, ACS Nano, 2016, 10, 93-101.

8 H. K. Moon, S. H. Lee and H. C. Choi, ACS Nano, 2009, 3, 3707-3713.

9 T. S. Hauck, T. L. Jennings, Y. Tetyana, J. C. Kumaradas and C. W. Chan, Adv. Mater., 2008, 20, 3832-3838.

10 J. Yu, D. Javier, M. A. Yaseen, N. Nitin, R. Richards-Kortum, B. Anvari, B. Anvari and M. S. Wong, J. Am. Chem. Soc., 2010, 132, 1929-1938.

11 Z.-H. Sheng, D.-H. Hu, M. B. Zheng, P.-F. Zhao, H.-L. Liu, D.-Y. Gao, P. Gong, G.-H. Gao, P.-F. Zhang, Y.-F. Ma and L.-T. Cai, ACS Nano, 2014, 8, 12310-12322.
12 B. R, Nat. Chem., 2011, 3, 570.

13 M. P. Rayman, Lancet, 2000, 356, 233-241.

14 J. E. Spallholz, L. M. Boylan and H. S. Larsen, Ann. N. Y. Acad. Sci., 1990, 587, 123-139.

15 X. Huang, X.-M. Liu, Q. Luo, J.-Q. Liu and J.-C. Shen, Chem. Soc. Rev., 2011, 40, 1171-1184.

16 E. N. Drake, Med. Hypotheses, 2006, 67, 318-322.

17 T. C. Stadtman, Annu. Rev. Biochem., 1996, 65, 83-100.

18 T.-Y. Li, M. Smet, W. Dehaen and H.-P. Xu, ACS Appl. Mater. Interfaces, 2016, 8, 3609-3614.

19 M. S. Stewart, J. E. Spallholz, K. H. Neldner and B. C. Pence, Free Radical Biol. Med., 1999, 26, 42-48.

20 X. Zhang, H.-P. Xu, Z.-Y. Dong, Y.-P. Wang, J.-Q. Liu and J.-C. Shen, J. Am. Chem. Soc., 2004, 126, 10556-10557.

$21 \mathrm{H}$. Tapiero, D. M. Townsend and K. D. Tew, Biomed. Pharmacother., 2003, 57, 134-144.

22 M. P. Rayman, Proc. Nutr. Soc., 2005, 64, 527-542.

23 Z.-Q. Deng, L.-L. Yu, W.-Q. Cao, W.-J. Zheng and T.-F. Chen, Chem. Commun., 2015, 51, 2637-2640.

24 P. B. Caffrey and G. D. Frenkel, Cancer Chemother. Pharmacol., 2000, 46, 74-78.

25 J. Kumi-Diaka, K. Merchant, A. Haces, V. Hormann and M. Johnson, J. Med. Food, 2010, 13, 842-850.

26 P. D. Whanger, Br. J. Nutr., 2004, 91, 11-28.

27 W.-T. Jiang, Y.-T. Fu, F. Yang, Y.-F. Yang, T. Liu, W.-J. Zheng, L.-L. Zeng and T.-F. Chen, ACS Appl. Mater. Interfaces, 2014, 6, 13738-13748.

28 L. Sarin, V. C. Sanchez, A.-H. Yan, A. B. Kane and R. H. Hurt, Adv. Mater., 2010, 22, 5207-5211.

29 S. Apostolou, J. O. Klein, Y. Mitsuuchi, J. N. Shetler, P. I. Poulikakos, S. C. Jhanwar, W. D. Kruger and J. R. Testa, Oncogene, 2004, 23, 5032-5040.

30 S. Misra, M. Boylan, A. Selvam, J. E. Spallholzm and M. Björnstedt, Nutrients, 2015, 7, 3536-3556.

31 H. E. Ganther, Biochemistry, 1968, 7, 2898-28905.

32 J. E. Spallholz, V. P. Palace and T. W. Reid, Biochem. Pharmacol., 2004, 67, 547-554.

33 J.-S. Zhang, X.-Y. Gao, L.-D. Zhang and Y.-P. Bao, BioFactors, 2001, 15, 27-38.

34 P. A. Tran, L. Sarin, R. H. Hurt and T. J. Webster, J. Biomed. Mater. Res., Part A, 2010, 93, 1417-1428.

35 Z. G. Gao, A. N. Lukyanov, A. Singhal and V. P. Torchilin, Nano Lett., 2002, 2, 979-982.

36 X.-H. Zheng, F.-F. Zhou, B.-Y. Wu, W. R. Chen and D. Xing, Mol. Pharm., 2012, 9, 514-522.

37 B. A. Graff, I. Bjornaes and E. K. Rofstad, Eur. J. Cancer, 2000, 36, 1433-1440.

38 E. I. Altinoğlu, T. J. Russin, J. M. Kaiser, B. M. Barth, P. C. Eklund, M. Kester and J. H. Adair, ACS Nano, 2008, 2, 2075-2084.

39 J.-L. Zhang, W. Jin, X.-Q. Wang, J.-C. Wang, X. Zhang and Q. Zhang, Mol. Pharm., 2010, 7, 1159-1168. 